\title{
A Multitone Analysis for Bioimpedance Spectroscopy using Minimal Digital Ressource
}

\author{
LIRMM \\ Univ. Montpellier, CNRS \\ Montpellier, France \\ soulier@lirmm.fr
}

Fabien Soulier, Achraf Lamlih, Vincent Kerzérho, Serge Bernard

\begin{abstract}
Bioimpedance spectroscopy consists in measuring the complex impedance of biological tissues over a large frequency domain. This method is convenient in particular for studying body composition, blood characterization and even cancer detection. This wide range of applications makes it suitable as a part of health monitoring systems. Today's self-monitoring devices tend to be portable, wearable or even implantable. Next generation bioimpedance sensing systems thus require to be implemented with power and resource savings in mind.

Impedance measurement methods are divided into two main categories. Some are based on "single-tone" signals while the others use "multi-tone" signals. First methods use a pure frequency sine wave to make the measurement. They benefit from a very simple analysis that may consist in synchronous demodulation. However, the operation must be repeated for each frequency over the domain of interest. Due to this necessary frequency sweep, the total measurement may take long. On the other hand, generating a multi-frequency signal allows the analysis to cover the whole frequency range simultaneously. This is at the cost of a more complex analysis algorithm (discrete cosine transform - DCT, typically). Unfortunately both methods result in excess power consumption: long time of measurement for single-tone frequency sweep, hardware and computational resources for multi-tone. That make both approaches hardly suitable for embedded applications.

In this paper, we propose an intermediate approach that combines the speed of multi-tone systems with a much simpler analysis algorithm than DCT or FFT. Using specific properties of the bioimpedance, we even show that we can get rid of any multiplier. This results in a minimal implementation using only adders and synchronous ADC. For optimal performances, this small footprint digital processing can be easily synthesized and embedded on a mixed-mode ASIC together with the analog frontend.
\end{abstract}

Index Terms-Bioimpedance spectroscopy, multi-frequency, digital processing

\section{INTRODUCTION}

Bioimpedance spectroscopy consists in measuring the complex impedance of biological tissues over a large frequency domain [1]. This method is convenient in particular for studying body composition [2], blood characterization [3] and even cancer detection [4]. This wide range of applications makes it suitable as a part of health monitoring systems. Today's self-monitoring devices tend to be portable, wearable or even

This work was supported by Ifremer (Popstar, Merlin project).

978-1-5386-5147-6/18/\$31.00 C 2018 IEEE implantable. Next generation bioimpedance sensing systems thus require to be implemented with power and resource savings in mind.

Impedance measurement methods are divided into two main categories. Some are based on "single-tone" signals while the others use "multi-tone" signals. First methods use a pure frequency sine wave to make the measurement [5], [6], [7]. They benefit from a very simple analysis that can consist in synchronous demodulation. However, the operation must be repeated for each frequency over the domain of interest. Due to this necessary frequency sweep, the total measurement may take long. On the other hand, generating a multi-frequency signal allows the analysis to cover the whole frequency range simultaneously [8], [9], [10]. This is at the cost of a more complex analysis algorithm (discrete cosine transform DCT, typically). Unfortunately both methods result in excess power consumption: long time of measurement for singletone frequency sweep, hardware and computational resources for multi-tone. That make both approaches hardly suitable for embedded applications.

In this paper, we propose an intermediate approach that combines the speed of multitone measurement with a much simpler analysis algorithm than DCT or FFT. First, we consider some a priori facts about bioimpedance. Those allow us to simplify the resolution needs for a bioimpedance-specific sensor compared to a generic purpose impedance analyzer. Based on this frequency distribution, we propose in the third section a simplified analysis process based on synchronous sampling demodulation. The fourth section gives the proposal of a digital implementation of the algorithm using only adders and frequency dividers. The last section opens some discussions about the limits of the proposed approach.

\section{BIOIMPEDANCE CHARACTERISTICS}

Let's put in evidence some general characteristics of bioimpedance. All biological tissues present similar frequency behaviors. The impedance is globally decreasing, with one or several relaxation domains [11], as we can see in Fig. 1.

It can be modeled with resistors, capacitors and so-called 

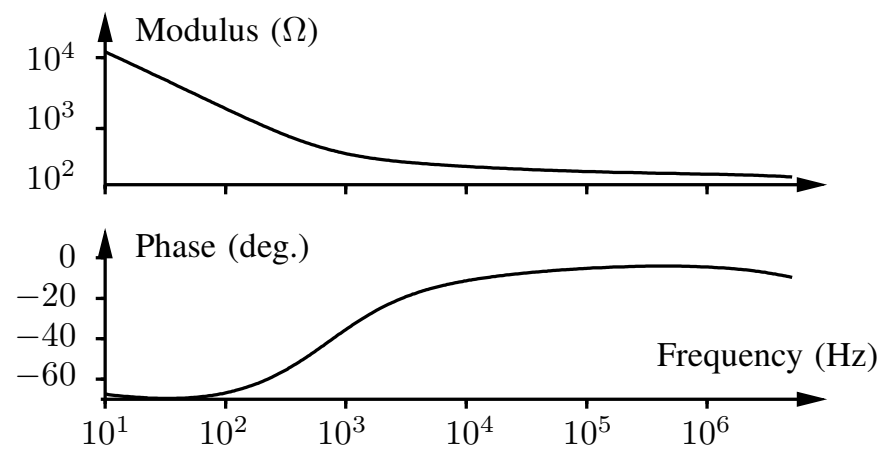

Fig. 1. Impedance of bluefin tuna muscle measured using a digital impedance analyzer (MFIA from Zurich Instruments AG).

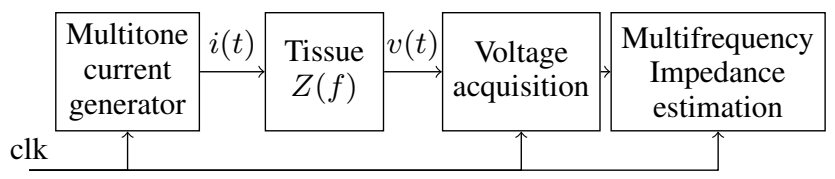

Fig. 2. Principle of multi-tone synchronous bioimpedance sensing system.

constant phase elements of impedance

$Z_{\mathrm{CPE}}(f)=\frac{1}{q_{0}(2 \mathrm{i} \pi f)^{\alpha}}, \quad q_{0}$ arbitrary parameter, $0<\alpha<1$.

Identifying such elements is better achieved with logarithmically growing frequencies. Designing a bioimpedance-specific sensor, we can adopt a frequency distribution of the form

$$
f_{i}=\frac{f_{0}}{2^{i}}, \quad 0 \leq i<n_{\mathrm{f}},
$$

for the multitone excitation signal. This give $n_{\mathrm{f}}$ frequencies with a maximum of $f_{0}$ and a resolution of $\log _{2}(10) \approx 3.32$ points per decade. This is expected to be enough to extract characteristics of quite smooth frequency responses.

\section{IMPEDANCE ESTIMATION}

The chosen principle of bioimpedance sensing is illustrated Fig. 2. With the above considerations, generated current $i(t)$ can be expressed as the superposition of $n_{\mathrm{f}}$ sine waves. Current being real, hermitian symmetry applies to complex amplitudes $\left(I_{-i}=I_{i}^{*}\right)$ such that

$$
i(t)=\sum_{i=0}^{n_{\mathrm{f}}-1} I_{i} \mathrm{e}^{2 \mathrm{i} \pi \frac{f_{0}}{2^{2}} t}+I_{i}^{*} \mathrm{e}^{-2 \mathrm{i} \pi \frac{f_{0}}{2^{i}} t} .
$$

For the sake of simplicity, we can let all $I_{i}$ to be equal to $\frac{1}{2}$ (normalized amplitudes and null phase for all frequency components). If not so, we can get back to this case by resistor calibration.

$$
i(t)=\frac{1}{2} \sum_{i=0}^{n_{\mathrm{f}}-1} \mathrm{e}^{2 \mathrm{i} \pi \frac{f_{0}}{2^{i}} t}+\mathrm{e}^{-2 \mathrm{i} \pi \frac{f_{0}}{2^{i}} t} .
$$

Let us express the Fourier transform $I(f)=\mathcal{F}\{i(t)\}$ in the frequency domain

$$
I(f)=\frac{1}{2} \sum_{i=0}^{n_{\mathrm{f}}-1} \delta\left(f-\frac{f_{0}}{2^{i}}\right)+\delta\left(f+\frac{f_{0}}{2^{i}}\right),
$$

with $\delta(t)$ denoting the Dirac distribution. We can deduce from the last equation the voltage expression $V(f)=\mathcal{F}\{v(t)\}$ in the sinusoidal steady state.

$$
\begin{aligned}
V(f) & =Z(f) I(f) \\
& =\frac{1}{2} \sum_{i=0}^{n_{\mathrm{f}}-1} Z_{i} \delta\left(f-\frac{f_{0}}{2^{i}}\right)+Z_{i}^{*} \delta\left(f+\frac{f_{0}}{2^{i}}\right),
\end{aligned}
$$

with $Z_{i}=Z\left(f_{i}\right)$ and $Z_{i}^{*}=Z\left(f_{-i}\right)$.

The objective of the following processing is to extract the values $Z_{i}=Z\left(f_{i}\right)$ from the acquired $v(t)$ signal. The main idea is to use synchronous sampling demodulation. Indeed, $Z\left(f_{0}\right)$ can be obtained by sampling $v(t)$ at the higher frequency $f_{0}$ and by low-pass filtering (averaging) the resulting samples. However, sampling the signal at lower frequencies results in aliasing. Let us explicitly express the result of sampling $v(t)$ at $f_{j}=\frac{f_{0}}{2^{j}}$. In the frequency domain, sampling is equivalent to convolution product by a Dirac comb.

$$
\begin{gathered}
V(f) * \sum_{k \in \mathbb{Z}} \delta\left(f-k \frac{f_{0}}{2^{j}}\right)= \\
\frac{1}{2} \sum_{k \in \mathbb{Z}} \sum_{i=0}^{n_{\mathrm{f}}-1} Z_{i} \delta\left(f-f_{0}\left(\frac{k}{2^{j}}+\frac{1}{2^{i}}\right)\right)+Z_{i}^{*} \delta\left(f-f_{0}\left(\frac{k}{2^{j}}-\frac{1}{2^{i}}\right)\right) .
\end{gathered}
$$

We isolate from the last equation the DC component $S_{j}$, i.e. the coefficient of $\delta(f)$. For all $i$ between 0 and $j$, there are two integers $k= \pm 2^{j-i}$ that satisfy

$$
\frac{1}{2^{i}} \pm \frac{k}{2^{j}}=0 .
$$

Summing all contributions, we get

$$
S_{j}=\frac{1}{2} \sum_{i=0}^{j}\left(Z_{i}+Z_{i}^{*}\right)=\sum_{i=0}^{j} \Re\left(Z_{i}\right) .
$$

In the same manner, we can get the quadrature components by delaying the sample time by a quarter of period $\frac{2^{j}}{4 f_{0}}$.

$$
\begin{gathered}
V(f) * \sum_{k \in \mathbb{Z}} \delta\left(f-k \frac{f_{0}}{2^{j}}\right) \mathrm{e}^{-2 \mathrm{i} \pi \frac{2^{j}}{4 f_{0}} f}= \\
\frac{1}{2} \sum_{k \in \mathbb{Z}} \sum_{i=0}^{n_{\mathrm{f}}-1} Z_{i} \delta\left(f-f_{0}\left(\frac{k}{2^{j}}+\frac{1}{2^{i}}\right)\right) \mathrm{e}^{-\mathrm{i} \pi \frac{k}{2}} \\
\quad+Z_{i}^{*} \delta\left(f-f_{0}\left(\frac{k}{2^{j}}-\frac{1}{2^{i}}\right)\right) \mathrm{e}^{-\mathrm{i} \pi \frac{k}{2}} .
\end{gathered}
$$

Again, for $k= \pm 2^{j-i}$ we get the DC component

$$
Q_{j}=\frac{1}{2} \sum_{i=0}^{j} Z_{i} \mathrm{e}^{-\mathrm{i} \pi \frac{-2^{j-i}}{2}}+Z_{i}^{*} \mathrm{e}^{-\mathrm{i} \pi \frac{2^{j-i}}{2}} .
$$




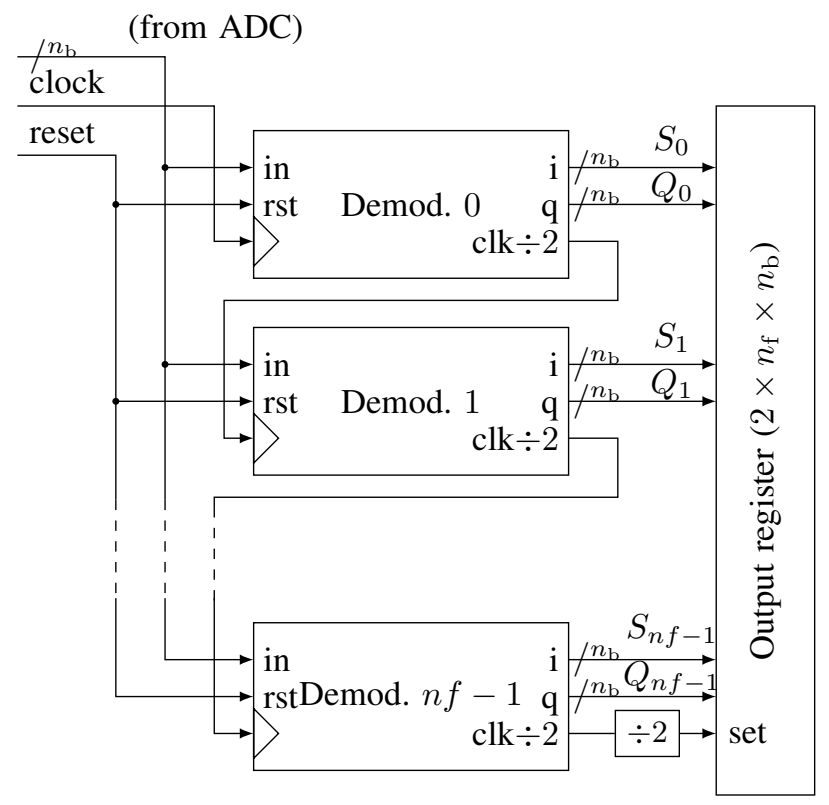

Fig. 3. Overview of the parallel implementation of the impedance estimation algorithm.

If $j \geq 2$, we can isolate the terms corresponding to $i=j$ and $i=j-1$.

$$
\begin{aligned}
Q_{j}= & \frac{1}{2} \sum_{i=0}^{j-2} Z_{i} \mathrm{e}^{\mathrm{i} \pi 2^{(j-i-1)}}+Z_{i}^{*} \mathrm{e}^{-\mathrm{i} \pi 2^{(j-i-1)}} \\
& +\frac{1}{2}\left(Z_{j-1} \mathrm{e}^{\mathrm{i} \pi}+Z_{j-1}^{*} \mathrm{e}^{-\mathrm{i} \pi}\right)+\frac{1}{2}\left(\mathrm{i} Z_{j}-\mathrm{i} Z_{j}^{*}\right)
\end{aligned}
$$

Thus,

$$
Q_{j}=\sum_{i=0}^{j-2} \Re\left(Z_{i}\right)-\Re\left(Z_{j-1}\right)-\Im\left(Z_{j}\right)
$$

From (10), (12) and (14), we can recursively calculate real and imaginary parts of $Z_{j}$.

$$
\begin{array}{ll}
\Re\left(Z_{0}\right)=S_{0}, & \Im\left(Z_{0}\right)=-Q_{0}, \\
\Re\left(Z_{1}\right)=S_{1}-S_{0}, & \Im\left(Z_{1}\right)=-Q_{1}-S_{0}, \\
\Re\left(Z_{j}\right)=S_{j}-S_{j-1}, & \Im\left(Z_{j}\right)=2 S_{j-2}-S_{j-1}-Q_{j} .
\end{array}
$$

\section{IMPLEMENTATION}

The main advantage of the proposed method is the simplicity of its digital implementation. Like classical synchronous demodulation, it only requires an ADC sharing the same clock as the signal generation (Fig. 2). Samples are fed by the ADC at the rate of $f_{\text {clk }}=4 f_{0}$. Then, they are re-sampled at each frequency $f_{i}$ composing the multitone signal. The extraction of the DC component can be easily done by averaging the samples over a period of the whole multitone signal. Then, sample decimation is performed between each stage by dividing the frequency by two (Fig. 3).

Practically, we These functions are grouped in a block called Demod, for demodulator, that can be easily instantiated from a generic description (Fig. 4). More in details, at each stage, we

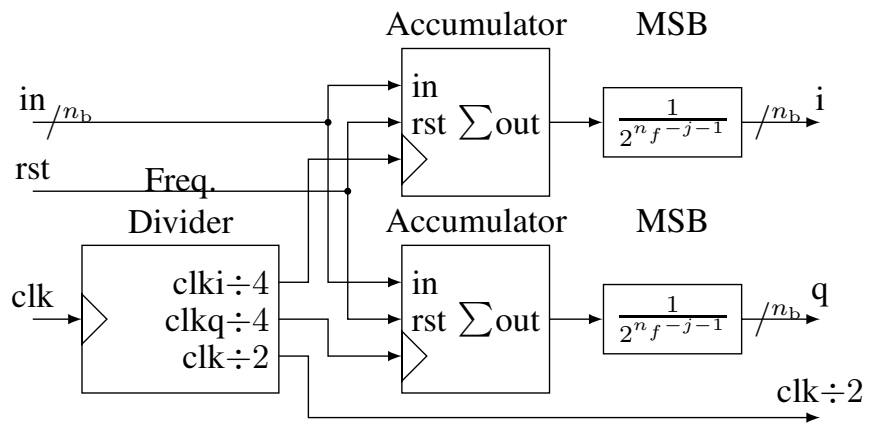

Fig. 4. Details of the "Demodulator" genreric block.

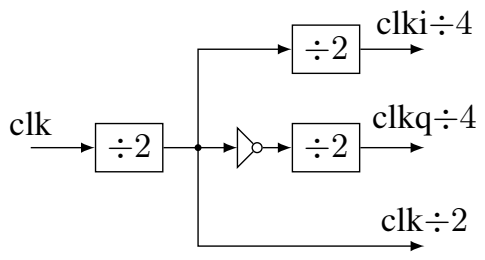

Fig. 5. Details of the "Freq. Divider" block.

generate two clock signals in quadrature (Fig. 5). They control two identical accumulators (Fig. 6). Samples are accumulated at the rate corresponding to a particular frequency $f_{i}$. The adder size is adapted to avoid overflow. Then dividing by $\frac{1}{2^{n} f^{-j-1}}$ is done by keeping only the $n_{b}$ most significant bits. So, all $S_{j}$ and $Q_{j}$ accumulation are eventually encoded with the same precision.

Values of $S_{j}$ (in-phase) and $Q_{j}$ (in-quadrature) are saved in the output register at the end of the global period $\left(\frac{2^{n_{\mathrm{f}}}}{f_{0}}\right)$. This is achieved using a simple frequency divider on the clock output of the last demodulator. We choose not to embed the computation of actual real and imaginary parts of the impedance as described by (15) to (17). It can be done without transmission penalty (same size of data) as a post-process. The objective is to keep the digital circuit as simple as possible in low-power constraint.

This parallel architecture is easily scalable. Moreover each stage only uses two adders together with a few flip-flops to divide the frequency. For optimal performances, it is totally realistic to embed this digital processing directly with the analog front-end (current source and sensing amplifier) in a mixed-mode ASIC.

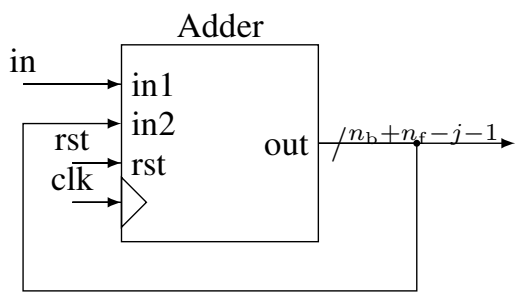

Fig. 6. Details of the "Accumulator" block. 


\section{DISCUSSION}

The proposed architecture may rise several questions. The main one should be the effect of noise on the measurement. We did not yet study in details the aliasing of white noise over the stages of the structure. In particular, low frequencies suffer from averaging low numbers of samples. The worst occurring for $f_{n_{\mathrm{f}}-1}=\frac{f_{0}}{2^{n_{\mathrm{f}}-1}}$ where only two samples are used to calculate $S_{\mathrm{f}}$ and $Q_{\mathrm{f}}$. However, we can observe that low frequencies have the benefit of best SNR while the acquisition, when using current generator and voltage sensor. This is due to the monotonically decreasing typical behavior of bioimpendances (see Fig. 1). For high frequencies where the SNR worsens, the averaging effect enhances the expected precision. Also, another possibility may obviously be the adaptation of current generation with increased amplitudes at high frequencies.

Another concern may be the very-low frequency noise due to amplifier offset, electronics $1 / f$ noise, electrode interface potential, etc. It would induce DC component that would impact all $S_{j}$ and $Q_{j}$ values. However, it appears relatively easy to get rid of this effect by averaging all samples at $f_{\text {clk }}$, i.e. before decimation and subtracting this DC value during post-process.

The last issue could be the fixed resolution due to frequency distribution as powers of $\frac{1}{2}$. If more resolution is needed, a non-power-of-two frequency division can be introduced, e.g. $\frac{f_{c l k}}{3}$ and then used instead of $f_{c l k}$ in a duplicated structure. Of course, expressions of $S_{j}$ and $Q_{j}$ will be affected and need to be re-evaluated. However, only post-processing computation will be modified.

\section{CONCLUSION}

We have proposed a very efficient implementation of multitone analysis for bioimpedance sensing. Thanks to general characteristics of bioimpedance, we have chosen a logarithmic frequency distribution. With this hypothesis, we can explicit the aliasing resulting from successive synchronous demodulations and decimations. This expression can be used to post-process the measurements and estimate real and imaginary parts of the impedance for each frequency. The digital implementation consists only in accumulators and frequency dividers and does not make any use of multipliers. Moreover, the structure can be easily scaled to wider frequency ranges.

\section{REFERENCES}

[1] S. Grimnes and O. G. Martinsen, Bioimpedance and Bioelectricity Basics. Academic Press, 2014

[2] D. Smith, M. Johnson, and T. Nagy, "Precision and accuracy of bioimpedance spectroscopy for determination of in vivo body composition in rats," International journal of body composition research, vol. 7, pp. 21-26, 2009.

[3] T. Dai and A. Adler, "In vivo blood characterization from bioimpedance spectroscopy of blood pooling," IEEE Transactions on Instrumentation and Measurement, vol. 58, no. 11, pp. 3831-3838, Nov 2009.

[4] P. Aberg, I. Nicander, J. Hansson, P. Geladi, U. Holmgren, and S. Ollmar, "Skin cancer identification using multifrequency electrical impedance-a potential screening tool," IEEE Transactions on Biomedical Engineering, vol. 51, no. 12, pp. 2097-2102, Dec 2004.

[5] S. Rodriguez, S. Ollmar, M. Waqar, and A. Rusu, "A batteryless sensor asic for implantable bio-impedance applications," IEEE Transactions on Biomedical Circuits and Systems, vol. 10, no. 3, pp. 533-544, June 2016.

[6] T. Chen, W. Wu, C. Wei, R. B. Darling, and B. Liu, "Novel 10bit impedance-to-digital converter for electrochemical impedance spectroscopy measurements," IEEE Transactions on Biomedical Circuits and Systems, vol. 11, no. 2, pp. 370-379, April 2017.

[7] AD5933 - 1 MSPS, 12-Bit Impedance Converter, Network Analyzer, Analog Devices, 2005, rev. F.

[8] B. Sanchez and R. Bragos, "Multifrequency simultaneous bioimpedance measurements using multitone burst signals for dynamic tissue characterization," Journal of Physics: Conference Series, vol. 224, no. 1, 2010.

[9] P. Kassanos and I. F. Triantis, "A cmos multi-sine signal generator for multi-frequency bioimpedance measurements," in 2014 IEEE International Symposium on Circuits and Systems (ISCAS), June 2014, pp. $249-252$.

[10] J. Ojarand, M. Rist, and M. Min, "Comparison of excitation signals and methods for a wideband bioimpedance measurement," Conference Record - IEEE Instrumentation and Measurement Technology Conference, vol. 2016-July, 2016.

[11] H. P. Schwan, "Electrical properties of tissue and cell suspensions," Advances in Biological and Medical Physics, vol. 5, pp. 147-209, 1957. 